(Aus der Universitäts - Frauenklinik in Gießen [Direktor: Prof. Dr. v. Jaschke].)

\title{
Der Einfluß von Proteinkörpern auf die Blutgerinnung.
}

Von

Dr. Rudolf Salomon und Dr. Ernst Vey,
Assistenten der Klinik.

(Eingegangen am 16. Mai 1922.)

Die Proteinkörpertherapie hat Umwandlungen in den Anschauungen verschiedener medizinischer Arbeitsgebiete hervorgerufen. Die spezifische Serumbehandlung geriet durch sie ins Wanken, Einflüsse auf das ganze Organsystem, auf den Gesamtstoffwechsel, auf die Tätigkeit endokriner Drüsen, auf die Temperatur usw. konnten sowohl bei gesunden wie bei kranken Individuen registriert werden. Heilwirkungen bei entzündlichen Veränderungen waren festzustellen, die Strahlentherapie bei malignen Neubildungen konnte durch sie unterstützt werden, wie die Untersuchungen von Opitz, v. Jaschke und Siegel ergaben. Uns galt es eine neue Komponente der Eiweißkörperwirkung zu ergründen: den Einfluß auf die Blutgerinnung.

Beobachtungen von Esch in der Zangemeister schen Klinik über günstige Erfahrungen mit den Proteinkörpern bei Behandlung von Gebärmutterblutungen, wonach bei reinen Menorrhagien sowohl die Blutungsstärke wie die Blutungsdauer vermindert werden konnte, ermunterten uns zu ausgedehnten experimentellen Nachuntersuchungen.

Der Weg, den wir dazu beschritten, gestaltete sich so, daß wir die im modernen Arzneischatz am meisten beobachteten Eiweißkörperrepräsentanten Caseosan und Aolan bioskopisch in Glasversuchen prüften. Da Weichardt, Starkenstein und Klingmüller mit anderen eiweißfreien Chemikalien ähnliche Grundwirkungen erzielen konnten, so wurden gleichzeitig einige in neuerer Zeit im Vordergrunde stehende Präparate in unseren Untersuchungskreis mit einbezogen. Wir. wählten Yatren, Placenta-Opton und Pituglandol. Nachdem wir den Einfluß dieser fünf Medikamente auf die Blutgerinnung extravasal geprüft hatten, wurden diese Substanzen verschiedenen Frauen einverleibt und darauf wieder die Veränderungen des Blutes in hämostatischer Hinsicht erforscht.

Caseosan zogen wir zu unseren Untersuchungen heran, weil wir bereits früher feststellen konnten, daß es sich um ein homogenes, chemisch 
konstantes Präparat handelt, daß sich therapeutisch günstig erwies. Aolan deshalb, weil Esch gerade damit, im Gegensatz zu Caseosan, einen besonderen Einfluß auf die Genitalblutungen beobachten konnte und ebenso E.F.Müller seine Einwirkung auf das hämostatische System, vor allem auf das Knochenmark, konstatierte. Xatren entfachte durch die interessanten Untersuchungen von Zimmer unsere Aufmerksamkeit. Ebenso Placenta-Opton, das von Abderhalden inauguriert ist und das nach Untersuchungen von $A$. Seitz einen Einflu $B$ auf die Blutungen in der Nachgeburtsperiode haben konnte; und schließlich prüften wir Pituglandol, welches in der modernen Geburtshilfe eine so große Rolle spielt.

An diese Versuche konnten wir auch deshalb herangehen, weil uns ein Gerinnungsapparat zur Verfügung stand, mit dem es möglich war, objektiv genaueste Vergleichsuntersuchungen anzustellen unter gleichartigen Bedingungen. Wir benutzen den Apparat von Bürker, der den großen Vorzug hat, daß die Temperatur, die auf die Gerinnungszeit von ausschlaggebender Bedeutung ist, sich völlig konstant erhalten läBt, daß man bei den zu untersuchenden Blutstropfen stets die gleiche Menge des Arzneimittels einwirken lassen kann.

Im ganzen wurden etwa 850 Versuche durchgeführt. Bei einer durchschnittlichen Temperatur von $25^{\circ} \mathrm{C}$ betrug die Gerinnungszeit beim gesunden Menschen ungefähr fünf Minuten; das ist der Zeitraum, den das Blut außerhalb des Gefäßes bis zum Auftreten des ersten Fibrinfadens durchläuft. Wir konnten dabei Bürkers Angabe bestätigen, daß die Gerinnungszeit beim Menschen normalerweise eine ziemlich konnstate ist und um fünf Minuten schwankt. In neuester Zeit konnte auch $P$. Reinewald gleiches beobachten. Als Ausgangsmaterial zu unseren Untersuchugen stellten sich Hebammenschülerinnen freiwillig zur Verfügung, ferner zogen wir Patientinnen heran, bei denen eine pathologische Organveränderung, welche auf die Gerinnung einen Einfluß hätte ausüben können, nicht nachweisbar war. Eine gewisse Abhängigkeit des Gerinnungsvorganges von der Tageszeit und Nahrungsaufnahme schalteten wir dadurch als, daßB die Untersuchungen möglichst zur selben Tagesstunde durchgeführt wurden.

Über die genauere Methodik ist in Bürkers Arbeit: ,Einfache Methode zur Bestimmung der Blutgerinnungszeit" nachzulesen, da wir uns in bezug auf die Anstellung der Versuche streng an die Vorschläge Bürkers hielten. Nur einige Punkte müssen noch besonders hervorgehoben werden. Um stets mit einem gleich großen Blutstropfen zu operieren, wurde den Fraven kurz vor der Blutentnahme ein kleiner Staustreifen um das zweite Phalangealgelenk angelegt; die Einstellung der Frankéschen Nadel zur Hautverletzung war stets die gleiche, was für die Exaktheit der Versuche uns wichtig dünkte. Ebenso wurde auch auf die stets gleiche Größe des Wassertropfens (bzw. Chemikalien) großes Gewicht 
gelegt, indem wir diesen Tropfen aus einer von uns besonders konstruierten, markierten Pipette ablaufen ließen. Auch das ist von großer Bedeutung. Denn nimmt man einen kleinen Tropfen Blut zu einem großen Wassertropfen, so wird infolge der starken Verdünnung die Gerinnungszeit verlängert werden können.

Als Mischflüssigkeit diente uns destilliertes, abgekochtes und wieder abgekühltes Wasser und wir fanden gleich Bürker, daß die Art der Flüssigkeit, ob Wasser oder physiologische Kochsalzlösung auf die Gerinnung ohne Einfluß ist. Mit den von uns experimenti causa angewandten Chemikalien verhält es sich ganz anders, wie unsere Untersuchungen noch erkennen lassen.

Sämtliche Blutentnahmen wurden aus der Fingerkuppe gemacht. Der Tropfen fiel frei ohne Ausbreitung auf der umgebenden Haut ohne Druck in die Mitte des hohlgeschliffenen Objektträgers, wodurch wir die Zeit von der Gewinnung des Tropfens bis zum Einbringen in den Apparat möglichst abkürzen und Fehlerquellen durch Abkühlen verhindern konnten. Nach Vermischung der beiden Tropfen haben wir die Suche nach einem Fibrinfaden alle halbe Minute vorgenommen, nicht häufiger, weil durch das öftere Hindurchfahren mit dem Glasstab eine Beeinflussung stattfindet, wodurch die Exaktheit der vergleichenden Untersuchungen leidet. Wichtig ist noch, daß die Fingerkuppe vor der Blutentnahme absolut trocken ist, und ihr kein Alkohol oder Aether mehr anhaftet, da diese die Gerinnung beschleunigen.

Zunächst galt es uns zu ergründen, wie sich die Gerinnungsbereitschaft verhält, wenn wir an Stelle von physiologischer Kochsalzlösung oder sterilem Wasser einen Proteinkörper auf das Blut einwirken ließen. Um nun gleichzeitig Vergleichswerte der verschiedenen Präparate zu bekommen, wurden die fünf eingangs erwähnten Chemikalien in verschiedenen Verdünnungen mit demselben Blute geprüft. So muBten oft bei einer Frau 30 und mehr Blutentnahmen gemacht werden. DaB die Beschaffung eines solchen Untersuchungsmaterials schwierig ist, dürfte verständlich sein.

Die Methodik gestaltete sich so, daß ein Tropfen des Reagen (z. B. Caseosan) auf das hohlgesehliffene Objektglas des Bürkerschen Apparates kam, und nun nach den oben angegebenen Regeln ein Blutstropfen dazu geträufelt wurde. Als Gerinnungszeit errechneten wir diejenige bis zum Nachweis des ersten Fadens. Des weiteren verfolgten wir noch die bald darauf einsetzende Klumpenbildung sowie die auftretende Totalgerinnung. Am wichtigsten für die Hämostase ist der erste Faden, da dieser rein objektiv abgelesen werden kann und auch von einer zuschauenden Hilfsperson in demselben Augenblick erkannt wird; während die Beurteilung der Klumpenbildung sowie der Gesamtgerinnung schon etwais Subjektives ist. Wir registrierten sie doch, weil wir 
ja nur Vergleichswerte zu kritischer Prüfung brauchten, und weil wir interessanterweise die Beobachtung machen konnten, daß die Präparate manchmal ohne Fadenbildung direkt eine Klumpenerzeugung oder sogar eine Totalgerinnung hervorriefen.

Aus der gleich folgenden Tab. I soll der Einfluß von Caseosan-Lindig auf das Blut von acht verschiedenen Frauen demonstriert werden. Zunächst ist die normale Gerinnung angegeben, dann folgt in der zweiten Kolumne die Wirkung des unverdünnten Präparates und dann reihen sich die Verdünnungszustände an $(1 / 2,1 / 10)$.

Tabelle $I$.

\begin{tabular}{|c|c|c|c|c|c|c|c|c|c|c|c|c|c|}
\hline \multirow{2}{*}{ 吾 } & \multicolumn{3}{|c|}{ Normale Gerinnung } & \multicolumn{3}{|c|}{ Unverdünnt } & \multicolumn{3}{|c|}{$1 / 8$ verdünnt } & \multicolumn{3}{|c|}{$1 / 10$ verdünnt } & \multirow{2}{*}{$\begin{array}{l}\text { Bem } \\
\text { kun! }\end{array}$} \\
\hline & Faden & Klump. & $\begin{array}{l}\text { Tot.- } \\
\text { Ger. }\end{array}$ & Faden & Klump. & $\begin{array}{l}\text { Tot. } \\
\text { Ger. }\end{array}$ & Faden & Klamp. & $\begin{array}{l}\text { Tot.- } \\
\text { Ger. } \\
\end{array}$ & Faden & Klump. & $\begin{array}{l}\text { Tot.- } \\
\text { Ger. }\end{array}$ & \\
\hline 1 & 6 & 7 & 9 & $-\infty$ & -4 & -7 & -1 & -1 & -3 & +2 & $(+-)$ & $(+-)$ & \\
\hline 2 & 5 & 6 & 10 & -3 & -3 & -2 & -1 & -3 & +1 & +1 & +1 & +3 & \\
\hline 3 & 5 & 7 & 8 & -6 & -9 & -12 & +2 & -1 & -3 & +1 & +1 & -1 & \\
\hline 4 & $3^{1 / 2}$ & 5 & 10 & -10 & -10 & -9 & -4 & -4 & -1 & $(+-)$ & +1 & +4 & \\
\hline$\check{5}$ & $3^{1 / 2}$ & $4^{1 / 2}$ & 6 & $-6^{1} / 2$ & $-71 / 2$ & -12 & $-1^{1 / 2}$ & $-21 / 2$ & -3 & $(+-)$ & $(+-)$ & -1 & \\
\hline 6 & 5 & $5 \frac{1 \%}{2}$ & $6^{1 / 2}$ & -3 & $-4^{1 / 2}$ & -6 & $+1 / 2$ & $(+-)$ & -1 & +2 & $+1 \frac{1}{2}$ & $+1^{1 / 2}$ & \\
\hline 7 & 5 & 6 & $71 / 2$ & $-3^{1 / 2}$ & $-4^{1 / 2}$ & $-81 / 2$ & $(1--)$ & -1 & $-1^{1 / 2}$ & $+1 / 2$ & $+1 / 2$ & $(+-)$ & \\
\hline 8 & $4^{1 / 2}$ & $5^{1 / 2}$ & 7 & $-8^{1} / 2$ & $-8^{1} / 2$ & -8 & $\mid-1$ & -1 & $-1^{1 / 2}$ & $-1 / 2$ & -1 & $(+-)$ & \\
\hline & 4,7 & 5,8 & 8 & $\left|\begin{array}{c}-5,8 \\
\text { (ollnne } \infty)\end{array}\right|$ & $-6,4$ & $-8,1$ & $-0,75$ & $-1,7$ & $-1,6$ & $+0,75$ & $+0,5$ & $+0,8$ & \begin{tabular}{|c} 
Mitt \\
wer
\end{tabular} \\
\hline
\end{tabular}

Zeichenerklärung (auch für die übrigen Zusammenstellungen):

$+=$ Beschleunigung der Gerinnung gegen die normale Gerinnung,

$-=$ Verzögerung $" n \quad n \quad n \quad n \quad n$

$(+-)=$ Übereinstimmung "mit der" normalen Gerinnung.

$\infty=$ Überhaupt keine Gerinnung.

Die Zahlen geben die Minuten an, um welche die Gerinnung beschleunigt bzw. verzögert wurde (nach Injektion der Chemikalien) gegen die normale Grerinnung der betreffenden Person.

Beim Überblicken dieser Zusammenstellung tritt die hohe Bedeutung der Konzentration des Präparates vor Augen. Ohne eine Ausnahme beweisen unsere Experimente, daß unverdünntes Caseosan im Glasversuch die Gerinnung hemmt. Mit steigender Verdünnung tritt ein Umschlag nach der entgegengesetzten Seite zutage. Bei zur Hälfte verdünntem Caseosan ist die Verzögerung lange nicht mehr so stark, ja bei zwei Fällen ( 3 und 6 ) schon gesteigert und auffallenderweise bei Fall 7 weder gehemmt noch beschleunigt, obwohl unverdünntes Caseosan eine starke Verzögerung der Gerinnung auslöste. Bei weiter zunehmender Verwässerung nahm die Gerinnungsherabsetzung weiter ab, und so beobachteten wir bei sieben von acht Frauen bei $1 / 10$ Verdünnungszustand eine Beschleunigung der Gerinnung. Bei der einen Ausnabme (Fall 8) war jedoch die Feststellung auffallend, daß bei unverdünntem 
Caseosan eine Verzögerung von 8,5 Minuten bestand, während bei $1 / 2$ Verdünnung eine solche von 1 Minute und bei $1 / 10$ Verdünnung nur noch eine solche von $1 / 2$ Minute vorhanden war. Errechnen wir aus unseren acht Fällen die Mittelwerte, so erkennen wir, daß es sich um Fälle mit normater Gerinnung handelt (Mittelwert 4,7 Minuten), und daß deren Blut bei Prüfung mit Caseosan in unverdünntem Zustande eine Verzögerung der Gerinnung aufwies (Mittelwert 5,8 Minuten), bei steigender Verwässerung die Hemmung immer mehr schwand (bei $1 / 2$ Verdünnung nur noch ein Mittelwert von $3 / 4$ Minute), und daß bei $1 / 10$ Verdünnung jetzt eine Beschleunigung der Gerinnung zu beobachten war (Mittelwert $3 / 4$ Minute). Die Klumpenbildung und Totalgerinnung verhielt sich ganz ähnlich.

Diese Feststellungen sind bedeutungsvoll, denn sie dürften uns Hinweise auf aie Wichtigkeit einer richtigen Dosierung der Proteinkörper und, wie wir noch sehen werden, auch der übrigen Chemikalien geben, denn bei höherer Konzentration haben wir eine diametral entgegengesetzte Wirkung wie bei niedrigem Gehalt. Damit dürften sich auch neue Wege zur Aufklärung der , Reizwirkung" ergeben.

Fin dem Caseosan ähnliches Verhalten zeigte Aolan in bezug auf die Blutgerinnung. Die beiden Präparate stimmten weitgehendst überein, was uns besonders interessierte, weil $E s c h$ mit Caseosan und mit Aolan so versehiedene klinische Beobachtungen in bezug auf das hämostatische System machte. Die klinische Bewertung der Proteinkörper auf das Blutsystem werden wir in einer anderen Abhandlung würdigen.

Tabelle II.

\begin{tabular}{|c|c|c|c|c|c|c|c|c|c|c|c|c|c|}
\hline \multirow{2}{*}{$\stackrel{2}{=}$} & \multicolumn{3}{|c|}{ Normale Gerinnung } & \multicolumn{3}{|c|}{ Unverdünnt } & \multicolumn{3}{|c|}{$1 / 2$ verdünnt } & \multicolumn{3}{|c|}{$1 / 10$ verdünnt } & \multirow{2}{*}{$\begin{array}{l}\text { Bemer- } \\
\text { kungen }\end{array}$} \\
\hline & Faden & Klump. & $\begin{array}{l}\text { Tot.- } \\
\text { Ger. }\end{array}$ & Faden & Klump. & $\begin{array}{l}\text { Tot.- } \\
\text { Ger. }\end{array}$ & Faden & Elump. & $\begin{array}{l}\text { Tot.- } \\
\text { Ger. }\end{array}$ & Faden & Klump. & $\begin{array}{l}\text { Tot.- } \\
\text { Ger. }\end{array}$ & \\
\hline 1 & 5 & $5^{1 / 2}$ & $6^{1} / 2$ & 0 & -5 & $-5^{1 / 2}$ & $+1 / 2$ & $(+-)$ & -1 & 11 & $+1 / 2$ & $(+-)$ & \\
\hline 2 & $4^{1 / 2}$ & $51 / 2$ & 7 & $-51 / 2$ & -6 & -5 & $-11 / 2$ & -1 & -1 & $-1 / 2$ & $(+-)$ & $(+-)$ & \\
\hline 3 & 5 & 6 & $7^{1} / 2$ & & & & $-1 / 2$ & $-1 / 2$ & $-1^{1 / 2}$ & +1 & +1 & $+1 / 2$ & \\
\hline 4 & 5 & $5^{1} / 2$ & $6^{1 / 2}$ & & -2 & $-21 / 2$ & +1 & $++^{-1 / 2}$ & $-1 / 2$ & $+1^{1 / 2}$ & $+1^{1 / 2}$ & $+1 / 2$ & \\
\hline 5 & $3^{1 / 2}$ & $4^{1 / 2}$ & 6 & -8 & $-81 / 2$ & -8 & & & & & & & \\
\hline 6 & $3^{1 / 2}$ & 5 & 10 & & $(+-)$ & +2 & -2 & & +2 & $-1 / 2$ & $+1 / 2$ & $+3^{1 / 2}$ & \\
\hline 7 & 5 & 7 & 8 & -3 & -3 & $-4^{1 / 2}$ & -2 & -2 & -3 & -1 & $(+-)$ & -2 & \\
\hline 8 & 5 & 6 & 10 & & $-\breve{5}$ & -4 & & $(+-)$ & $(+-)$ & & +1 & +2 & \\
\hline 9 & 6 & 7 & 9 & $-\infty$ & $(+-)$ & $(+-)$ & $(+-)$ & -1 & $+1 / 2$ & +1 & -3 & -2 & \\
\hline & 4,9 & 5,7 & 7,3 & $-5,4$ & $-4,2$ & $-3,9$ & $-0,75$ & $-0,67$ & $-0,75$ & $+0,36$ & $+0,25$ & $+0,42$ & $\mid \begin{array}{c}\text { Mittel- } \\
\text { werte }\end{array}$ \\
\hline
\end{tabular}

Genau wie bei Caseosan war auch bei Aolan in unverdïnntem $\mathrm{Zu}$ stande eine Verzögerung der Gerinnung in sämtlichen Fällen nachweisbar, und ließ sich als Mittelwert 5,4 Minuten errechnen, für Caseosan 5,8 Minuten; so daß also beide Präparate in den Zahlen fast überein- 
stimmen. Wurde Aolan zur Hälfte verdünnt, so orgab sich als Mittelwert dieselbe Zahl wie bei Caseosan (-0,75 Minute); bei 1/10 Gehalt trat der Umschlag von der Gerinnungsverzögerung zur Steigerung der Gerinnungsfähigkeit ein (-0,36 Minute), nicht ganz die Höhe von Caseosan erreichend.

Aolan ist demnach ein Eiweißkörper, der in konzentriertem Zustande gerinnungshemmend, mit steigender Verdünnung gerinnungstördernd im Glasversuche wirht. Also auch bei Aolan dürfte die richtige Dosierung bei therapeutischer Anwendung von großem Finflusse sein.

DaB das Yatren, das in chirurgischen Kreisen in neuerer Zeit viel verwandt wird, in seiner Wirkung von der richtigen Dosierung abhängigist, dürften unsere Untersuchungen ebenfalls ergeben. Die Tab. III soll dies erläutern.

Tabelle III.

\begin{tabular}{|c|c|c|c|c|c|c|c|c|c|c|c|c|c|}
\hline \multirow{2}{*}{ ! } & \multicolumn{3}{|c|}{ Normale Gerinnung } & \multicolumn{3}{|c|}{ Unverdünnt } & \multicolumn{3}{|c|}{$1 / 2$ verdünnt } & \multicolumn{3}{|c|}{$1 / 10$ verdünnt } & \multirow{2}{*}{$\begin{array}{l}\text { Bemer } \\
\text { kungen }\end{array}$} \\
\hline & Faden & Klump. & $\begin{array}{l}\text { Tot.. } \\
\text { Ger. }\end{array}$ & Faden & Klump. & $\begin{array}{l}\text { Tot.- } \\
\text { Ger. }\end{array}$ & Faden & Klump. & $\begin{array}{l}\text { Tot.- } \\
\text { Ger. }\end{array}$ & Faden & Klump. & $\begin{array}{l}\text { Tot.- } \\
\text { Ger. }\end{array}$ & \\
\hline 1 & 6 & 7 & 9 & $-\infty$ & $-\infty$ & $-\infty$ & $-\infty$ & $-\infty$ & $-\infty$ & +2 & +2 & +3 & \\
\hline 2 & 5 & 6 & 10 & $-\infty$ & $-\infty$ & $-\infty$ & -4 & & & -1 & -2 & $-\infty$ & \\
\hline 3 & 5 & 7 & 8 & $-\infty$ & $-\infty$ & $-\infty$ & -3 & & & $(+-)$ & -1 & & \\
\hline 4 & $3^{1} / 2$ & 5 & 10 & $-\infty$ & $-\infty$ & $-\infty$ & $-8^{1 / 2}$ & $-81 / 2$ & -8 & -4 & -4 & -1 & \\
\hline 5 & $3^{1 / 2}$ & $4^{1 / 2}$ & 6 & $-\infty$ & $-\infty$ & $-\infty$ & $-\infty$ & $-\infty$ & $-\infty$ & $-21 / 2$ & $-2^{1 / 2}$ & -3 & \\
\hline 6 & 5 & $5^{1 / 2}$ & $61 / 2$ & $-\infty$ & $-\infty$ & $-\infty$ & -4 & $-\infty$ & & $+31 / 2$ & +3 & $+21 / 2$ & \\
\hline 7 & 5 & 6 & $7 / 2$ & $-\infty$ & $-\infty$ & $-\infty$ & $-1^{1 / 2}$ & $-11 / 2$ & -1 & $-1 / 2$ & $-1 / 2$ & $-1 / 2$ & \\
\hline 8 & $4^{1} / 2$ & $5^{1 / 2}$ & 7 & $-\infty$ & $-\infty$ & $-\infty$ & -4 & & & -2 & $-1^{1 / 2} \mid 2$ & $-1^{1 / 2}$ & \\
\hline 9 & 5 & $51 / 2$ & $6^{1 / 2}$ & $-\infty$ & $-\infty$ & $-\infty$ & $(+-)$ & $(+-)$ & $-3 / 4$ & -2 & -3 & -4 & \\
\hline & 4,9 & 5,7 & 7,3 & $-\infty$ & $-\infty$ & $-\infty$ & $\begin{array}{l}-4,2 \\
(\infty \text { un } \\
\text { sichtigt) }\end{array}$ & $\begin{array}{l}-\infty \\
\text { berück- } \\
2 \times \infty\end{array}$ & $-\infty$ & $-0,7$ & $-0,7$ & $-0,6$ & $\begin{array}{l}\text { Mittel- } \\
\text { werte }\end{array}$ \\
\hline
\end{tabular}

Unverdünntes Yatren bewirkte eine vollkommene Hemmung der Blutgerinnung; das Blut blieb dünnflüssig; es kam auch nicht einmal zur Klumpenbildung. Bei Verwendung von zur Hälfte verdünntem Yatren war die Hämostase zwar noch stark verzögert, jedoch in den meisten Fällen jetzt in meßbaren Grenzen. Bei einer Person (9) war keine Verzögerung gegen die Norm nachweisbar und bei Fall 7 nur eine geringe Herabminderung.

Mit zunehmender Verwässerung nahm die Gerinnungshemmungsfähigkeit weiter $a b$, so daß in zwei Fällen sogar eine Beschleunigung sich einstellte (1,6 Minute); bei einem weiteren Fall (3) zeigte sich normales Verhalten und bei einer anderen Frau ( 7 ) war die Gerinnung in dem $1 / 10$ verdünntem Zustande nur noch um eine halbe Minute verzögert. Interessant ist die Kurve, in welcher sich die errechneten Mittelwerte bewegen : bei unverdünntem Yatren, von $\infty \mathrm{zu}-4$ Minuten $(1 / 2$ verdünnt) bis $\mathrm{zu}-0,67$ Minute bei $1 / 10$ Verdünnung. 
Ähnliche Eigenschaften wie die Proteinkörper zeigte in bezug auf die Gerinnnungsfähigkeit des Blutes das von Merck hergestellte PlacentaOpton. Hier erkennen wir wieder das Parallelgehen der steigenden Verdünnung des Präparates mit der Beschleunigung des Blutgerinnungsvermögens. Jedoch verzögerte Placenta-Opton in unverdünntem $\mathrm{Zu}$ stande die Gerinnung weit weniger wie die bis jetzt besprochenen Körper; wir konnten bei vier Individuen $(1,3,10,11)$ sogar eine Gerinnungsförderung feststellen. Schon bei $50 \%$ iger Verdünnung ließ sich in den meisten Fällen eine Beschleunigung erkennen, die bei zunehmender Verwässerung $(1 / 10)$ eine weitere Anschwellung erfuhr. Vergleichen wir die Mittelwerte der elf untersuchten Frauen, so errechnet sich für unverdünntes PlacentaOpton eineGerinnungsverzögerung von 1,1 Minute, bei $1 / 2$ Verdünnung eine Beschleunigung von -0,1 Minute; bei $1 / 10$ eine Beschleunigung von 1,3 Minuten. Besonders scharf reagierten in dieser Hinsicht zwei gravide Frauen $(10,11)$.

Tabelle $I V$.

\begin{tabular}{|c|c|c|c|c|c|c|c|c|c|c|c|c|c|}
\hline \multirow{2}{*}{$\stackrel{0}{=}$} & \multicolumn{3}{|c|}{ Normale Gerinnung } & \multicolumn{3}{|c|}{ Unverdünnt } & \multicolumn{3}{|c|}{$1 / 2$ verdünnt } & \multicolumn{3}{|c|}{$1 /$ vo verdünnt } & \multirow{2}{*}{$\begin{array}{l}\text { Bemer- } \\
\text { kungen }\end{array}$} \\
\hline & Faden & Klump. & $\begin{array}{l}\text { Tot.- } \\
\text { Ger. }\end{array}$ & Faden & Klump. & $\begin{array}{l}\text { Tot.- } \\
\text { Ger. }\end{array}$ & Faden & Klump. & $\begin{array}{l}\text { Tot.- } \\
\text { Ger. }\end{array}$ & Faden & Klump. & $\begin{array}{l}\text { Tot.- } \\
\text { Ger. }\end{array}$ & \\
\hline 1 & 5 & 7 & 8 & +1 & +1 & +1 & +2 & +3 & +2 & +3 & +4 & +2 & \\
\hline 2 & 6 & 7 & 9 & +1 & +1 & +1 & +2 & -1 & -3 & +4 & +2 & +3 & \\
\hline 3 & 5 & 6 & 10 & -1 & -1 & +2 & +1 & $+1^{1} / 2$ & +4 & +2 & +2 & +4 & \\
\hline 4 & $31 / 2$ & $4^{I} / 2$ & 6 & $-2^{1 / 2}$ & -3 & -3 & $-1 / 2$ & $-1 / 2$ & -1 & $-1 / 2$ & $(+-)$ & $\left.(t-)_{1}\right)$ & \\
\hline 5 & $31 / 2$ & 5 & 10 & -3 & -2 & $(\div-)$ & -1 & $(+-)$ & +4 & $-1 / 2$ & $+1 / 2$ & +5 & \\
\hline 6 & 5 & $5^{1 / 2}$ & $6^{1 / 2}$ & -6 & $-81 / 2$ & $-81 / 2$ & $(+-)$ & $-1 / 2$ & $+1 / 2$ & $+1^{1 / 2}$ & $+1 \frac{1}{2}$ & $+11 / 2$ & \\
\hline 7 & 5 & $5^{1 / 2}$ & $6^{1} / 2$ & $-2^{1} / 2$ & $-4^{1 / 2}$ & & -1 & -3 & -3 & $-11 / \mathrm{a}$ & $-11 / 2$ & -2 & \\
\hline 8 & 5 & 6 & $71 / 2$ & -1 & -2 & -3 & $+11 / 2$ & +1 & -2 & +2 & $+1^{1 / 2}$ & $+1 / 2$ & \\
\hline 9 & $4^{1 / 2}$ & $5^{1 / 2}$ & 7 & $-1 / 2$ & $(+-)$ & +1 & $-6^{1} / 2$ & -6 & -6 & $-1^{1 / 2}$ & $-11 / 2$ & -1 & \\
\hline 10 & 6 & & 9 & +1 & & +1 & +2 & & -3 & +4 & & +3 & \\
\hline 11 & 5 & & 8 & +1 & & +1 & +2 & & +2 & +2 & & +2 & \\
\hline & 4,8 & 5,8 & 7,9 & $-1,1$ & $-2,1$ & $-0,9$ & $+0,1$ & -06 & $-0,5$ & $+1,3$ & $+0,9$ & $+1,6$ & $\begin{array}{c}\text { Mittel- } \\
\text { werte }\end{array}$ \\
\hline
\end{tabular}

Unser besonderes Interesse beansprucht dann noch Pituglandol, das in der Austreibungsperiode stark wehenerregend und damit auf die Uterusmuskulatur kontrahierend wirkt, wodurch ein Einfluß auf die Vasomotoren ausgeübt wird, was zur Verminderung der Blutung führt. Könnte es nicht möglich sein, daß diesen Präparaten selbst eine blutgerinnende Eigenschaft innewohnt? Unsere extravasalen Glasversuche lenken die Aufmerksamkeit nach dieser Richtung hin. Während wir (wie die folgende Tab. V zeigt) bei Pituglandol in unverdünntem oder $1 / 2$ verdünntem Zustande eine Verzögerung der Gerinnung feststellen mußten, trat bei $1 / 10$ Verdünnung ein deutlicher Umsehlag nach der entgegengesetzten Seite ein und damit eine Gerinnungsbeschleunigung wie Tab. V zeigt. 
Tabelle $\mathrm{V}$.

\begin{tabular}{|c|c|c|c|c|c|c|c|c|c|c|c|c|c|}
\hline \multirow{2}{*}{ 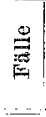 } & \multicolumn{3}{|c|}{ Normale Gerinnung } & \multicolumn{3}{|c|}{ Unverdünnt } & \multicolumn{3}{|c|}{ 1/2 verdünnt } & \multicolumn{3}{|c|}{$1 / 10$ verdünnt } & \multirow{2}{*}{$\begin{array}{l}\text { Beme } \\
\text { kunge }\end{array}$} \\
\hline & Faden & Elump. & $\begin{array}{l}\text { Tot.- } \\
\text { Ger. }\end{array}$ & Faden & Klump. & $\begin{array}{l}\text { Tot.- } \\
\text { Ger. }\end{array}$ & Faden & Klump. & $\begin{array}{c}\text { Tot,- } \\
\text { Ger. }\end{array}$ & Faden & Klump. & $\begin{array}{l}\text { Tot:- } \\
\text { Ger. }\end{array}$ & \\
\hline 1 & 5 & 6 & 10 & -3 & -4 & -4 & $+1 / 2$ & -1 & $+1 / 2$ & +1 & +1 & +3 & \\
\hline 2 & 6 & 7 & 9 & & & & & -4 & -3 & +3 & +3 & +4 & \\
\hline 3 & $3^{1} / 2$ & 5 & 10 & -4 & -4 & -1 & $-1^{1 / 2}$ & -2 & +1 & $-11 / 2$ & $-1 / 2$ & $+31 / 2$ & \\
\hline 4 & 5 & 7 & 8 & -6 & - & - & $(+-)$ & -1 & -2 & +1 & +2 & +1 & \\
\hline 5 & $3^{1} / 2$ & $4^{1 / 2}$ & 6 & $+1^{1 / 2}$ & +2 & +2 & & & & & & & \\
\hline 6 & 5 & 6 & $71 / 2$ & $(t-)$ & -1 & -2 & $-1 / 2$ & $-1 / 2$ & $\left(+\frac{7}{5}-\right)$ & $+1 / 2$ & $+3 / 4$ & +1 & \\
\hline 7 & $\bar{n}$ & $51 / 2$ & $6^{1 / 2}$ & -2 & $-3^{1} / 2$ & $-4^{1 / 2}$ & $-1 / 2$ & $-1 \frac{1}{2}$ & $-11 \%$ & $+1 / 4$ & $+1 / 4$ & +1 & \\
\hline 8 & $4^{1 / 2}$ & $51 / 2$ & 7 & $+21 / 2$ & +3 & +3 & $-1 / 2$ & $-1 / 2$ & & $+1^{1 / 2}$ & $+11 / 2$ & $+1 / 2$ & \\
\hline
\end{tabular}

Ein Vergleich dieser fünt untersuchten Präparate läßt also erkennen, daß sie die gemeinschaftliche Eigenschaft haben, in unverdünntem Zustande gerinnungshemmend zu wirken, mit steigender Verdünnung auch eine Steigerung der Gerinnungstähigkeit des Blutes auszulösen.

Die weitesten Ausschläge in dieser Hinsicht ergaben Yatren, dann folgten die EiweiBkörper Caseosan, Aolan sowie Placenta-Opton und Pituglandol.

Über weitere Vergleichsuntersuchungen dieser verschiedenen Chemikalien sollen die folgenden Úbersichtsbilder Aufschluß geben. Da ja individuelle Blutdifferenzen bestehen könnten, so wurden die fünf Medikamente nacheinander auf das Blut derselben Person geprüft, womit wirklich genaue Vergleichsskalen geschaffen wurden. Unsere Untersuchungsreihen beliefen sich auf neun Frauen. Da die häufigen Blutentziehungen den Versuchspersonen nicht sehr angenehm sind, so ist die Materialbeschaffung nicht ganz einfach. Wir verwandten zum großen Teil unsere Hebammenschülerinnen.

Die folgende Tabelle illustriert, daß die Präparate Yatren und Caseosan in unverdünntem Zustande bei derselben Frau das Blut am stärksten beeinflussen, so daß bei Yatren nach einer halben Stunde noch keine Spur Gerinnung nachweisbar war; bei Caseosan war dieselbe

Tabelle VI. Fall Obermaier.

\begin{tabular}{|c|c|c|c|c|c|c|c|c|c|c|c|c|c|}
\hline & \multicolumn{3}{|c|}{$\begin{array}{c}\text { Normale } \\
\text { Gerinnung }\end{array}$} & \multicolumn{3}{|c|}{$\begin{array}{l}\text { Einflus der unver- } \\
\text { dünnten Chemik. }\end{array}$} & \multicolumn{3}{|c|}{$\begin{array}{l}\text { Einfluß } 1 / 2 \text { ver- } \\
\text { dïnnter Chemik. }\end{array}$} & \multicolumn{3}{|c|}{$\begin{array}{l}\text { EinfluB } 1 / 1 a \text { ver- } \\
\text { dünnter Chemikalien }\end{array}$} & \multirow[b]{2}{*}{ Bemerkungen } \\
\hline & 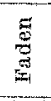 & $\dot{\hat{g}}$ & 荬 & 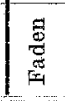 & $\dot{m}$ & 妾通 & 恋 & 啇 & 这 & 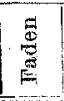 & 啇 & 荅宫 & \\
\hline $\begin{array}{l}\text { Caseosan . } \\
\text { Aolan ... } \\
\text { Yatren. . } \\
\text { Plac.-Opton. } \\
\text { Pituglandol. }\end{array}$ & 6 & 7 & 9 & $\mid \begin{array}{c}- \\
\infty \\
+1 \\
- \\
\text { (Nich }\end{array}$ & $\begin{array}{l}-6 \\
+- \\
\infty \\
+1 \\
-1\end{array}$ & $\begin{array}{c}-10 \\
+- \\
\infty \\
+1 \\
- \\
-\end{array}$ & $\left|\begin{array}{c}-1 \\
+- \\
\infty \\
+2\end{array}\right|$ & $\left|\begin{array}{c}-1 \\
-1 \\
\infty \\
-1 \\
-3\end{array}\right|$ & $\begin{array}{c}-3 \\
+1 / 2 \\
\infty \\
-3 \\
-3\end{array}$ & $\begin{array}{l}+2 \\
+1 \\
+2 \\
+4 \\
+3\end{array}$ & $\begin{array}{c}(+-) \\
-3 \\
+2 \\
+2 \\
+3\end{array}$ & $\begin{array}{l}-4 \\
+2^{1 / 2} \\
+3 \\
+4\end{array}$ & $\begin{array}{c}\text { Bel Vollcaseosan } \\
\text { Kein Fibrinfade1 } \\
\text { desgl. }\end{array}$ \\
\hline
\end{tabular}


stark verzögert und ließ sich überhaupt keine Fadenbildung beobachten, sondern sofort Klumpen. Bei Aolan war die Reaktion schon geringer; sie unterschied sich nur von der normalen physiologischen Gerinnung dadurch, daß ebenfalls keine Faden-, sondern direkt klumpige Gerinnung bedingt wurde. Einen Gegensatz zu diesen Substanzen bildete Placenta-Opton, das bereits in unverdünntem Zustande eine Beschleunigung der Hämostase um eine Minute hervorrief.

Bei derselben Patientin behielt Yatren in $1 / 2$ verdünntem Zustande die gleiche Eigenschaft bei; während Aolan auch jetzt kaum eine Veränderung veranlaßte, wogegen Caseosan schon eine Steigerung der Gerinnung feststellen ließ. Placenta-Opton bewirkte eine noch mehr zutage tretende Gerinnungsbeschleunigung, dagegen fiel die Verzögerung der Klumpenbildung und Totalgerinnung auf. Pituglandol zeigte auch jetzt noch keine starke Verzögerung der Gerinnung.

Wurden die Präparate auf $1 / 10$ verdünnt, so lösten sämtliche eine Beschleunigung des Gerinnungsvermögens aus, am auffallendsten in die Augen springend bei Placenta-Opton, dann folgten Pituglandol, Yatren, Caseosan und Aolan.

Die folgenden 8 Fälle können wir nur in Skizzen erläutern, da die Kürze, welche die Abhandlung einnehmen muß, uns eine weitere text-

Tabelle VII. Fall Strauß.

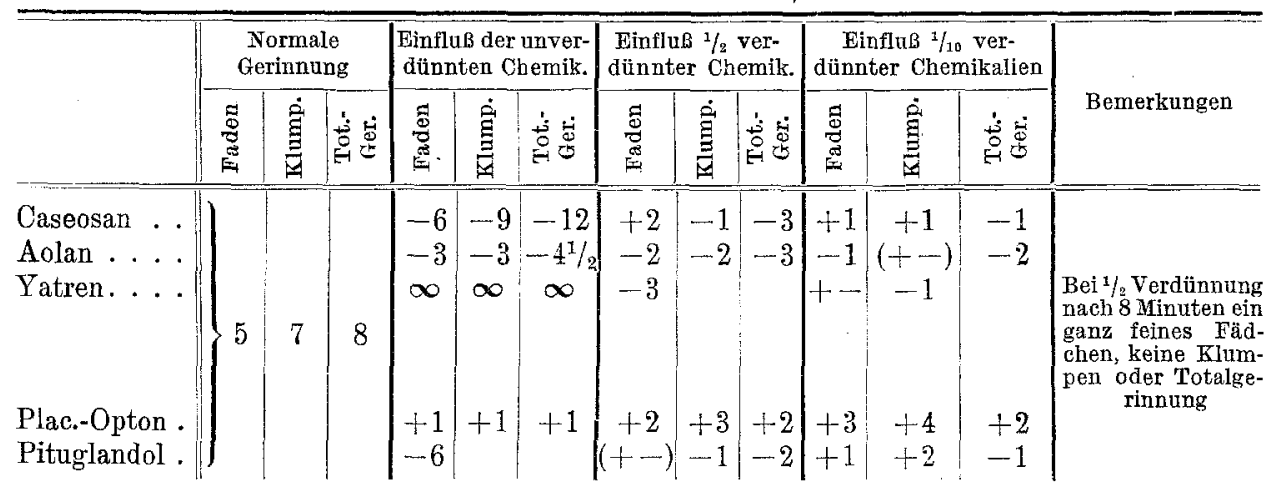

Tabelle VIII. Fall Bink.

\begin{tabular}{|c|c|c|c|c|c|c|c|c|c|c|c|c|c|}
\hline & \multicolumn{3}{|c|}{$\begin{array}{l}\text { Normale } \\
\text { Gerinnung }\end{array}$} & \multicolumn{3}{|c|}{$\begin{array}{l}\text { Einfluß der unver- } \\
\text { dünnten Chemikalien }\end{array}$} & \multicolumn{3}{|c|}{$\begin{array}{c}\text { Einflu } B 1 / 2 \text { verdünnter } \\
\text { Chemikalien }\end{array}$} & \multicolumn{3}{|c|}{$\begin{array}{c}\text { Einfluß } 1 / 10 \text { verdünnter } \\
\text { Chemikalien }\end{array}$} & \multirow{2}{*}{$\begin{array}{l}\text { Bemer- } \\
\text { kungen }\end{array}$} \\
\hline & 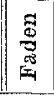 & $\underset{\dot{g}}{g}$ & 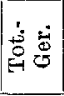 & 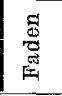 & Klump. & $\begin{array}{l}\text { Tot.- } \\
\text { Ger. }\end{array}$ & Faden & Klump. & $\begin{array}{l}\text { Tot.- } \\
\text { Ger. }\end{array}$ & Faden & Klump. & $\begin{array}{l}\text { Tot.- } \\
\text { Ger. }\end{array}$ & \\
\hline $\begin{array}{l}\text { Caseosan . } \\
\text { Aolan ... } \\
\text { Yatren. . . } \\
\text { Plac.-Opton. } \\
\text { Pituglandol . }\end{array}$ & 5 & $5^{1 / 2}$ & $6^{1} / 2$ & $\begin{array}{l}-\infty \\
-6 \\
-2\end{array}$ & $\mid \begin{array}{l}-4^{1 / 2} \\
-2 \\
-\infty \\
-8^{1 / 2} \\
-3^{1} / 2\end{array}$ & $\left|\begin{array}{l}-6 \\
-21 / 2 \\
-\infty \\
-8^{1} / 2 \\
-4^{1 / 2}\end{array}\right|$ & $\left|\begin{array}{c}+^{1 / 2} \\
+1 \\
-4 \\
\left(+-\frac{1}{-1 / 2}\right.\end{array}\right|$ & $\mid \begin{array}{l}(+-) \\
+1 / 2 \\
-\infty \\
-1 / 2 \\
-1^{1} / 2\end{array}$ & $\begin{array}{l}-1 \\
-1 / 2 \\
-\infty \\
+1 / 2 \\
-1 / 2\end{array}$ & $\begin{array}{l}+2 \\
+1^{1 / 2} \\
+3^{y / 2} \\
+1^{1 / 2} \\
+1 / 4\end{array}$ & $\left|\begin{array}{l}+11 / 2 \\
+11 / 2 \\
+3 \\
+11 / 2 \\
+1 / 4\end{array}\right|$ & $\begin{array}{l}+11 / 2 \\
+1 / 2 \\
+2^{1 / 2} \\
+1^{1 / 2} \\
+1\end{array}$ & 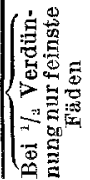 \\
\hline
\end{tabular}


Tabelle IX. Fall Steinebach.

\begin{tabular}{|c|c|c|c|c|c|c|c|c|c|c|c|c|c|}
\hline & \multicolumn{3}{|c|}{$\begin{array}{l}\text { Normale } \\
\text { Gerinnung }\end{array}$} & \multicolumn{3}{|c|}{$\begin{array}{l}\text { Einfluß der unver- } \\
\text { dünnten Chemikalien }\end{array}$} & \multicolumn{3}{|c|}{$\begin{array}{l}\text { Einflud } 1 / 2 \text { verduinnter } \\
\text { Chemikalien. }\end{array}$} & \multicolumn{3}{|c|}{$\begin{array}{l}\text { Einflub } 1 / 10 \text { ver- } \\
\text { dünnter Chemikalien }\end{array}$} & \multirow{2}{*}{ 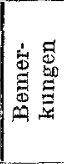 } \\
\hline & 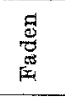 & 寞 & 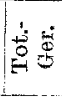 & Faden & Klump. & $\begin{array}{l}\text { Tot.- } \\
\text { Ger. }\end{array}$ & Faden & Klump. & $\begin{array}{l}\text { Tot. } \\
\text { Ger. }\end{array}$ & Eaden & Klump. & $\begin{array}{l}\text { Tot.- } \\
\text { Ger. }\end{array}$ & \\
\hline $\begin{array}{l}\text { Aolan .... } \\
\text { Yatren. . . } \\
\text { Plac.-Opton. } \\
\text { Pituglandol. }\end{array}$ & $3^{1 / 2}$ & $4^{1} / 2$ & 6 & $\begin{array}{c}-6^{1 / 2} \\
-8 \\
\infty \\
-21 / 2 \\
+11 / 2\end{array}$ & $\mid \begin{array}{c}-7^{1 / 2} \\
-8^{1} / 2 \\
\infty \\
-3 \\
+2\end{array}$ & $\begin{array}{c}-12 \\
-8 \\
\infty \\
-3 \\
+2\end{array}$ & $\begin{array}{c}-1^{1 / 2} \\
-\infty \\
-1 / 2\end{array}$ & $\left|\begin{array}{c}-21 / 2 \\
-\infty \\
-1 / 2\end{array}\right|$ & $\begin{array}{c}-3 \\
-\infty \\
-1\end{array} \mid$ & $\left|\begin{array}{l}-1 / 2 \\
-21 / 2 \\
-1 / 2\end{array}\right|$ & $\begin{array}{l}-1 / 2 \\
-21 / 2 \\
(+-)\end{array} \mid$ & $\begin{array}{c}-1 \\
-3 \\
(+--)\end{array} \mid$ & \\
\hline
\end{tabular}

Tabelle $X$. Fall Adrienne.

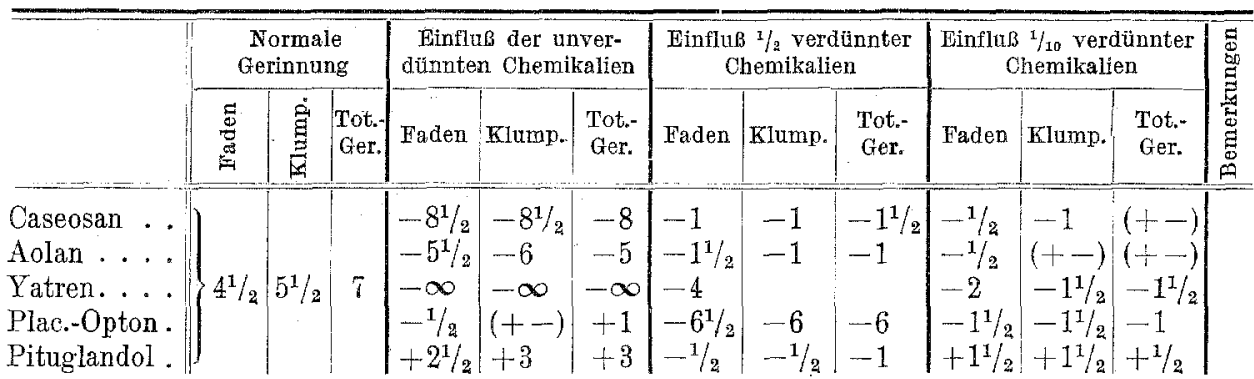

Tabelle XI. Fall Fischer.

\begin{tabular}{|c|c|c|c|c|c|c|c|c|c|c|c|c|c|}
\hline & \multicolumn{3}{|c|}{$\begin{array}{c}\text { Normale } \\
\text { Gerinnung }\end{array}$} & \multicolumn{3}{|c|}{$\begin{array}{l}\text { Einflus der unver- } \\
\text { dünnten Chemikalien }\end{array}$} & \multicolumn{3}{|c|}{$\begin{array}{c}\text { Einfluß } 1 / 2 \text { verdünnter } \\
\text { Chemikalien }\end{array}$} & \multicolumn{3}{|c|}{$\begin{array}{c}\text { Einfluß } 1 / 10 \text { verdünn } \\
\text { ter Chemikalien }\end{array}$} & \multirow{2}{*}{$\begin{array}{l}\text { Be- } \\
\text { merkungen }\end{array}$} \\
\hline & 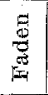 & : & 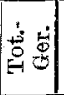 & Faden & Klump. & $\begin{array}{l}\text { Tot.- } \\
\text { Ger. }\end{array}$ & Faden & Klump. & $\begin{array}{l}\text { Tot.- } \\
\text { Ger. }\end{array}$ & $\begin{array}{l}\text { Fa- } \\
\text { den }\end{array}$ & Klump. & $\begin{array}{l}\text { Tot:- } \\
\text { Ger. }\end{array}$ & \\
\hline $\begin{array}{l}\text { Oaseosan . } \\
\text { Aolan . . } \\
\text { Yatren. . }\end{array}$ & 5 & 6 & 10 & $\begin{array}{c}-3^{1 / 2} \\
\infty\end{array}$ & $\begin{array}{c}-4^{1} / 2 \\
-5 \\
\infty\end{array}$ & $\begin{array}{c}-1 / 2 \\
-4 \\
\infty\end{array}$ & $\begin{array}{l}-1 \\
-4\end{array}$ & $\begin{array}{l}-3 \\
-1 \\
-1\end{array}$ & $\begin{array}{c}+1 \\
(+-)\end{array}$ & $\mid \begin{array}{l}+1 \\
-1\end{array}$ & $\left|\begin{array}{c}+1 \\
(+-) \\
-2\end{array}\right|$ & $\begin{array}{l}+3 \\
+2\end{array}$ & $\begin{array}{l}\text { Bei } 1 / 2 \text { Dosis } \\
\text { nach } 9^{\prime} \text { feines } \\
\text { Fädchen. Bei } \\
1 / 10 \text { Verdün- } \\
\text { nung keine To- } \\
\text { talgerinnung. }\end{array}$ \\
\hline $\begin{array}{l}\text { Plac-Opton } \\
\text { Pituglandol }\end{array}$ & & & & $\begin{array}{l}-1 \\
-3\end{array}$ & $\begin{array}{l}-1 \\
-4\end{array}$ & $\begin{array}{l}-2 \\
-4\end{array}$ & $\left|\begin{array}{l}+1 \\
+1 / 2\end{array}\right|$ & $\left|\begin{array}{l}+1 / 2 \\
-1\end{array}\right|$ & $\begin{array}{l}+4 \\
+1 / 2\end{array}$ & $\begin{array}{l}+2 \\
+1\end{array}$ & $\begin{array}{l}+2 \\
+1\end{array}$ & $\left|\begin{array}{l}+4 \\
+3\end{array}\right|$ & \\
\hline
\end{tabular}

Tabelle XII. Fall Nebel.

\begin{tabular}{|c|c|c|c|c|c|c|c|c|c|c|c|c|c|}
\hline & \multicolumn{3}{|c|}{$\begin{array}{l}\text { Normale } \\
\text { Gerinnung }\end{array}$} & \multicolumn{3}{|c|}{$\begin{array}{l}\text { Einflub der unver- } \\
\text { dünnten Ohemikalien }\end{array}$} & \multicolumn{3}{|c|}{$\begin{array}{c}\text { Einflus } 1 / 2 \text { verdünnter } \\
\text { Chemikalien }\end{array}$} & \multicolumn{3}{|c|}{$\begin{array}{l}\text { Einfluß }{ }^{1 / 10} \text { verdünnter } \\
\text { Chemikalien }\end{array}$} & \\
\hline & $\underbrace{8}_{\mathbb{8}}$ & 寞 & 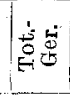 & Faden & Klump. & $\begin{array}{l}\text { Tot.- } \\
\text { Ger. }\end{array}$ & Faden & Klump. & $\begin{array}{l}\text { Tot.- } \\
\text { Ger. }\end{array}$ & Faden & Klump. & $\begin{array}{l}\text { Tot.- } \\
\text { Ger. }\end{array}$ & \\
\hline $\begin{array}{l}\text { Caseosan . } \\
\text { Aolan ... . } \\
\text { Yatren. . . } \\
\text { Plac.-Opton . } \\
\text { tuglandol . }\end{array}$ & 5 & $5^{1 / 2}$ & $6^{1 / 2}$ & $\left|\begin{array}{l}-5 \\
-\infty \\
-2^{1 / 2}\end{array}\right|$ & $\mid \begin{array}{l}-5 \\
-\infty \\
-4^{1} / 2 \\
\text { Nicht }\end{array}$ & $\begin{array}{l}-5^{1 / 2} \\
-\infty \\
\text { durchg }\end{array}$ & $\left|\begin{array}{l}(+-) \\
+1 / 2 \\
(+-) \\
-1\end{array}\right|$ & $\left|\begin{array}{c}-1 / 2 \\
(+-) \\
(+-) \\
-3 \\
\text { da Pat }\end{array}\right|$ & $\mid \begin{array}{c}-1 \\
-1 \\
-3 / 4 \\
-3 \\
\text { tient } \mathrm{s}\end{array}$ & $\begin{array}{l}+1 \\
-2 \\
-1^{1} / 2 \\
\text { sich we }\end{array}$ & $\begin{array}{c}+1 / 2 \\
-3 \\
-11 / 2 \\
\text { igert! }\end{array}$ & $\left|\begin{array}{l}(+-) \\
-4 \\
-2\end{array}\right|$ & \\
\hline
\end{tabular}


Tabelle XIII. Fall Konrad.

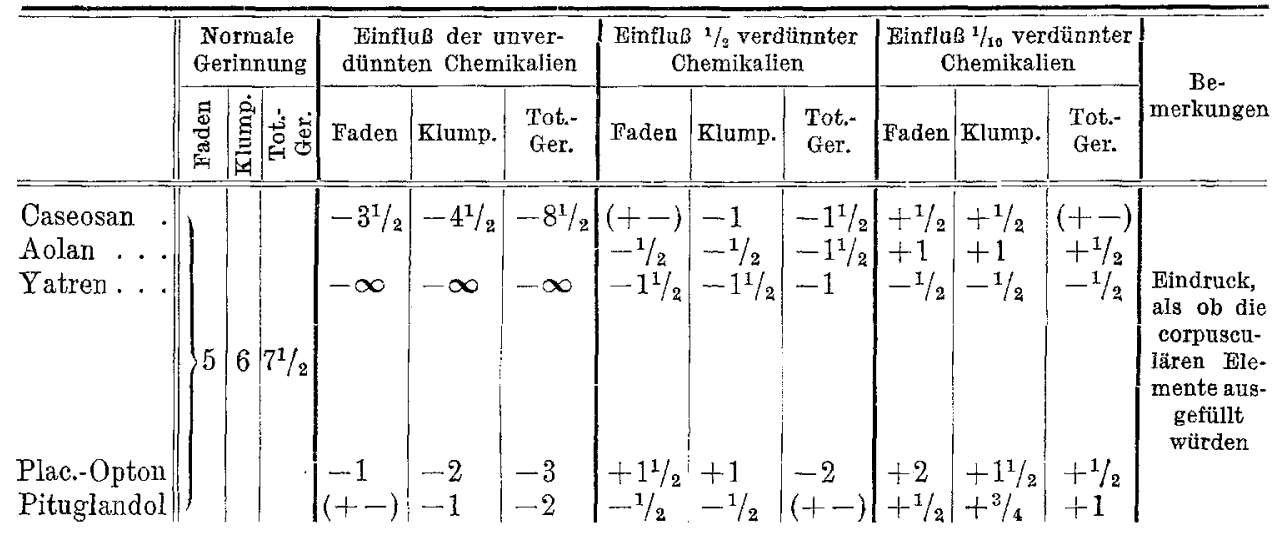

Tabelle XIV. Fall Rube.

\begin{tabular}{|c|c|c|c|c|c|c|c|c|c|c|c|c|c|}
\hline & \multicolumn{3}{|c|}{$\begin{array}{l}\text { Normale } \\
\text { Gerinnung }\end{array}$} & \multicolumn{3}{|c|}{$\begin{array}{c}\text { Einfluß unverdünnter } \\
\text { Chemikalien }\end{array}$} & \multicolumn{3}{|c|}{$\begin{array}{l}\text { Einflu } \beta^{1 / 2} \text { verduinnter } \\
\text { Chemikalien }\end{array}$} & \multicolumn{3}{|c|}{$\begin{array}{c}\text { Einfluß } 1 / 10 \text { verdünnter } \\
\text { Chemikalien }\end{array}$} & \multirow{2}{*}{$\begin{array}{c}\mathrm{Be}- \\
\text { merkungen }\end{array}$} \\
\hline & 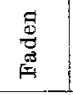 & 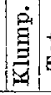 & 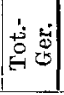 & Faden & 离 & $\begin{array}{l}\text { Tot.- } \\
\text { Ger. }\end{array}$ & Faden & Klump. & $\begin{array}{l}\text { Tot.- } \\
\text { Ger. }\end{array}$ & Faden & Elump. & $\begin{array}{l}\text { Tot.- } \\
\text { Ger. }\end{array}$ & \\
\hline $\begin{array}{l}\text { Caseosan } \\
\text { Aolan . . } \\
\text { Yatren . . } \\
\text { Plac.-Opton } \\
\text { Pituglandol }\end{array}$ & $3^{1 / 2}$ & 5 & 10 & $\begin{array}{c}-10^{1} / 2 \\
\infty \\
-3 \\
-4\end{array}$ & $\begin{array}{l}-10 \\
+2 \\
\infty \\
-2 \\
-4\end{array}$ & $\begin{array}{c}-9 \\
+2 \\
\infty \\
(+-) \\
-1\end{array}$ & $\left|\begin{array}{l}-4 \\
-2 \\
-8^{1} / 2 \\
-1 \\
-2^{1 / 2}\end{array}\right|$ & $\left|\begin{array}{l}-4 \\
-81 / 2 \\
(+-) \\
-2\end{array}\right|$ & $\begin{array}{r}-1 \\
+2 \\
-8 \\
+4 \\
+1\end{array}$ & $\begin{array}{l}(+-) \\
-1 / 2 \\
-4 \\
-1 / 2 \\
-11 / 2\end{array}$ & $\begin{array}{l}+1 \\
+1 / 2 \\
-4 \\
+1 / 2 \\
-1 / 2\end{array}$ & $\begin{array}{l}+3^{1 / 2} \\
-3^{1 / 2} \\
-1 \\
+5 \\
+3^{1} / 2\end{array}$ & 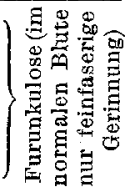 \\
\hline
\end{tabular}

liche Ausdehnung verbietet. Wir lassen die übrigen Versuchsresultate in tabellarischer Übersicht folgen. Daraus läßt sich die bereits eingangs erwähnte GesetzmäBigkeit deutlich herauslesen und auch der Einfluß der Individuen auf die verschiedenen Pröparate.

Ermuntert durch die Erfahrungen dieser experimentellen Untersuchungen wandten wir uns rein klinischen Beobachtungen in bezug auf die Blutgerinnung zu. Nachdem unsere Versuche einen einwandfreien Einfluß der Proteinkörper auf das Gerinnungsvermögen offenbart hatten, injizierten wir Eiweißsubstanzen Patientinnen und prüften erneut die Wechselbezichungen im hämostatischen System. Von den mit Proteinen angestellten Versuchen sollen heute nur unsere Ergebnisse mit Caseosan besprochen werden, da wir die Erfahrung machten, daß Aolan viele übereinstimmende Erscheinungen zeitigte. Wahrscheinlich hätten wir bei Verwendung irgendwelcher anderer Eiweißkörper, wie Milch usw., ähnliche Resultate erzielen können. Darüber hoffen wir demnächst ausführlicher zu berichten. Fier galt es uns nur auf neue Gesichtspunkte in der Proteinkörperbehandlung aufmerksam zu machen und zu ausgedehnten Nachuntersuchungen anzuregen. 

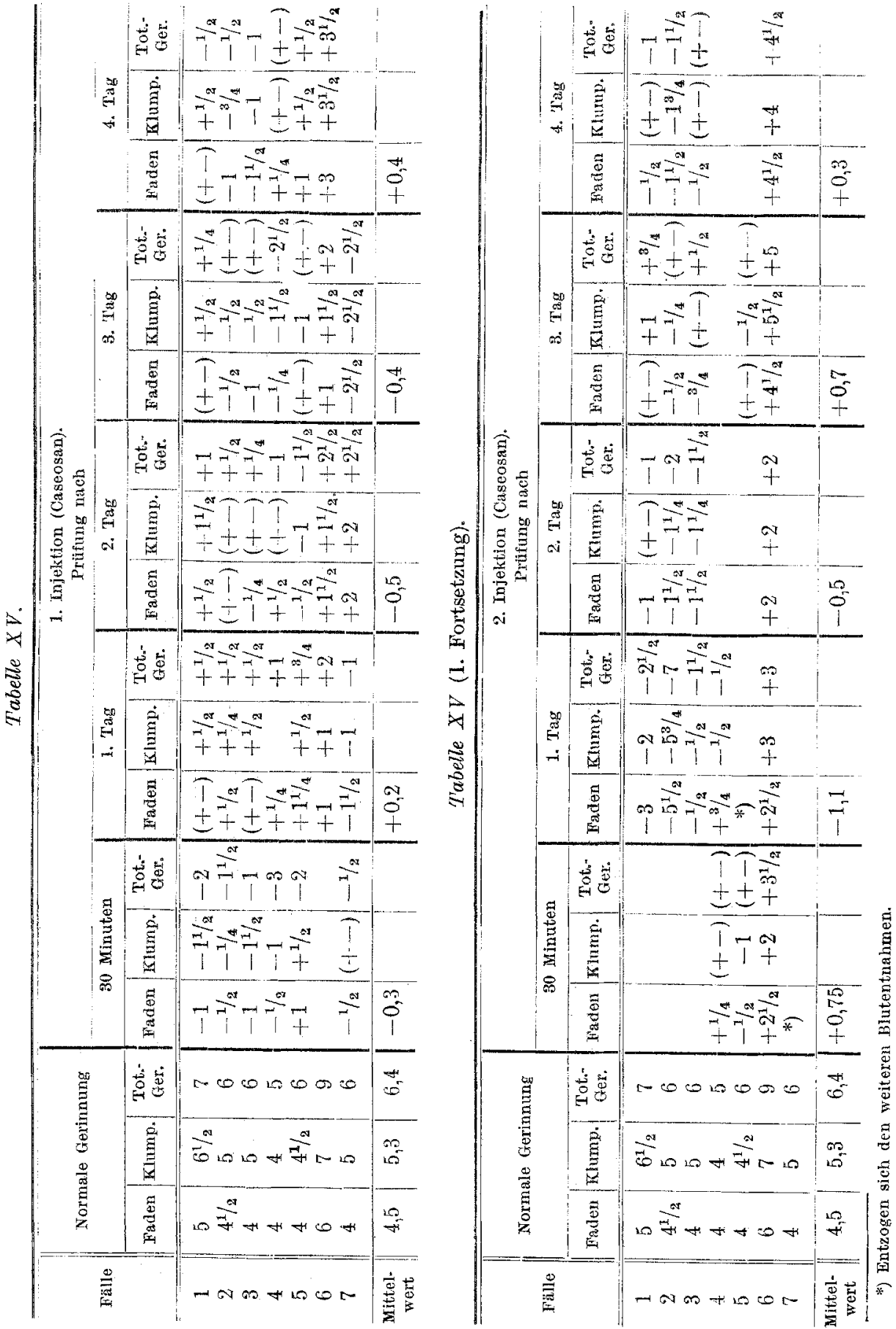


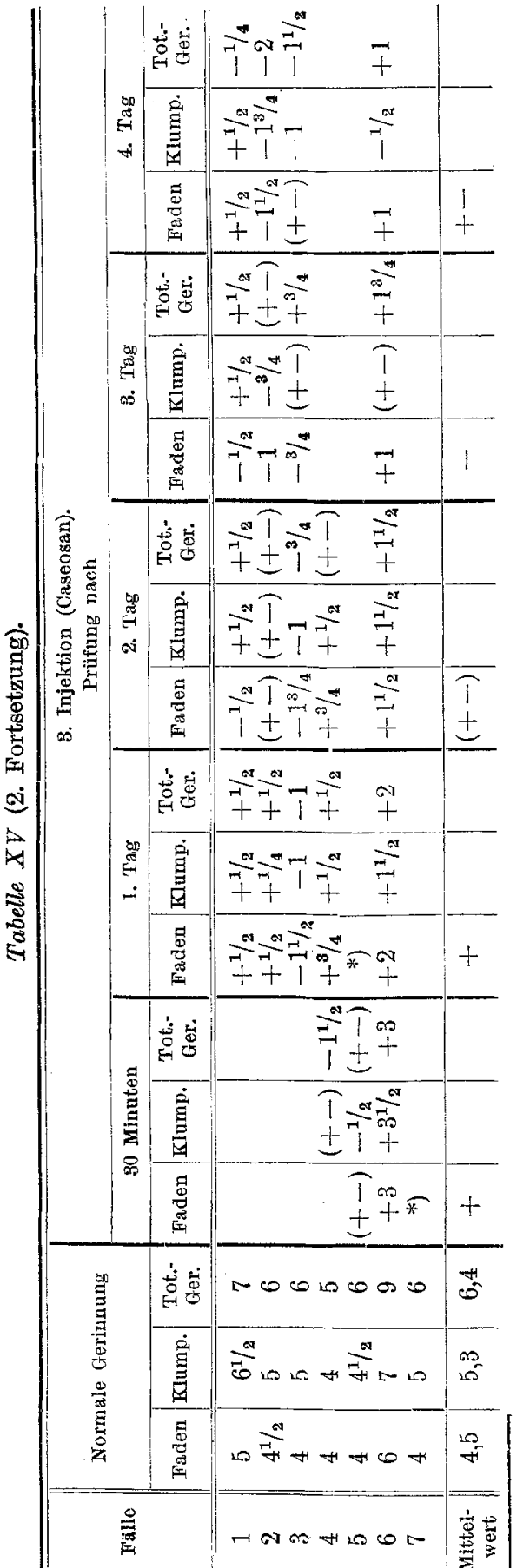

Wenn wir nun die Wirkung von Caseosan genauer beleuchten, so finden wir, daß bei dér Einverleibung desselben intravenös oder intramuskulär schon bei Dosen von $1 / 2 \mathrm{ccm}$ sich nach 30 Minuten bereits ein Einfluß auf die Gerinnung des Blutes bemerkbar machte, und zwar zunächst in verzögerndem Sinne (5 von 6 Fällen). Über das Verhalten der Gerinnungsform, betreffend Klumpenbildung, und der Gesamtgerinnung geben die Tabellen Auskunft. - Auch in den folgenden Tagen war noch ein Einfluß auf das Gerinnungsvermögen vorhanden, und wir verfolgten es vier Tage lang. Auffallend war, daß in den nächsten Tagen keine Hemmung der Hämostase, sondern im Gegenteil eine geringe Beschleunigung zu beobachten war (außer am dritten Tage, wo die Verzögerungen über die Beschleunigungen überwiegten).

Nach der zweiten Injektion, die fünf Tage nach der ersten gemacht wurde, ließ sich keine Konstanz im Verhalten registrieren. Man gewann den Eindruck, als ob plötzlich wieder eine Hemmung zutage treten wollte; während sich unmittelbar nach der dritten Applikation wieder eine Beschleunigung der Gerinnung einstellte.

Kurz zusammenfassend läßt sich also sagen, daß bei einer geringen Injektionsmenge zunächst eine Verzögenung der Gerinnung auflritt, daß jedoch nach 
mehreren Applikationen sich eine Beschleunigung konstatieren läßt. Freststehend ist, daß die Einverleibung van Eiweißsubstanzen in den Organismus einen Einfluß auf die Gerinnungsfähigkeit des Blutes ausübt. Unsere Untersuchungsreihen sind noch zu klein, um bindende Schlüsse zu erlauben.

Von den übrigen Präparaten sollen nur noch die Ergebnisse, die sich mit Placenta-Opton erzielen ließen, geschildert werden. Es wurde so verIahren, da $B$ in stündlichen Intervallen dreimal hintereinander je $1 \mathrm{ccm}$ Placenta-Opton intramuskulär appliziert und darauf das Gerinnungsverhalten geprüft wurde.

Tabelle XVI. Placenta-Opton.

\begin{tabular}{|c|c|c|c|c|c|c|c|c|c|c|c|c|c|}
\hline & & erinn & & $\begin{array}{r}\mathrm{B}] \\
30^{\prime} \mathrm{n}\end{array}$ & a & ztion & $\begin{array}{l}\text { Blv } \\
30^{\prime} \text { na }\end{array}$ & 2. & tion & $\begin{array}{r}\mathrm{Bl} \\
30^{\prime} \mathrm{n}\end{array}$ & 15 & ke & \\
\hline & $\frac{\vec{\pi}}{\mathrm{z}}$ & 苞 & 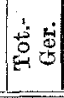 & Faden & Klump. & $\begin{array}{l}\text { Tot.- } \\
\text { Ger. }\end{array}$ & Faden & Klump. & $\begin{array}{l}\text { Tot:- } \\
\text { Ger. }\end{array}$ & Faden & Klump. & $\begin{array}{l}\text { Tot.- } \\
\text { Ger. }\end{array}$ & Fälle \\
\hline $\begin{array}{r}1 \\
2 \\
3 \\
4 \\
5 \\
6 \\
7 \\
8 \\
9 \\
10\end{array}$ & \begin{tabular}{|l}
$21 / 2$ \\
$33 / 4$ \\
4 \\
5 \\
5 \\
$4^{1 / 2}$ \\
4 \\
4 \\
4 \\
$4^{1} / 2$
\end{tabular} & $\begin{array}{l}31 / 2 \\
4 \\
4^{1 / 2} \\
6 \\
6 \\
5^{1 / 2} \\
5^{1 / 2} \\
5 \\
4^{1 / 2}\end{array}$ & \begin{tabular}{|l|}
6 \\
5 \\
6 \\
$7^{1} / 2$ \\
9 \\
6 \\
$71 / 2$ \\
6 \\
$5^{1} / 2$ \\
$7^{1} / 2$
\end{tabular} & $\begin{array}{l}-1 \\
+{ }^{3} / 4 \\
+1 \\
+2 \\
+2 \\
+1 \\
+1 \\
+1 / 2 \\
+1 \\
+1^{1 / 2}\end{array}$ & $\begin{array}{l}-3 / 2 \\
-1 \\
-1 / 2 \\
+2 \\
+2^{1 / 2} \\
+1 \\
+2 \\
+1 / 2 \\
(+-)\end{array}$ & $\begin{array}{l}+1 \\
-1 \\
(+-) \\
+11 / 2 \\
+3 \\
(+-) \\
+11 / 2 \\
+1 \\
+1 / 2 \\
+11 / 2\end{array}$ & $\mid \begin{array}{l}+1 \\
+11 / 2 \\
+1 \\
+1 \\
-1 / 2 \\
(+--) \\
+1 / 2 \\
+1^{1 / 2}\end{array}$ & $\begin{array}{l}-11 / 2 \\
-1 / 4 \\
(+-1) \\
+1 \\
+1 \\
+1 \\
+^{3} / 4 \\
+1 / 4\end{array}$ & $\begin{array}{l}(+-) \\
+1 / 2 \\
+1 / 2 \\
+2 \\
+3 \\
(+-) \\
+1 \\
+1 \\
-1 / 2 \\
+2\end{array}$ & $\begin{array}{l}-1 / 4 \\
+1 \\
+1^{1 / 2} \\
+1^{1 / 2} \\
+2 \\
-2 \\
(+-) \\
(+-) \\
+1^{1 / 2}\end{array}$ & $\begin{array}{l}+1 / 2 \\
-1 \\
+1 / 2 \\
+1^{1 / 2} \\
+2 \\
-1 \\
(+-) \\
-1 / 2\end{array}$ & $\begin{array}{l}(+-) \\
+1 \\
+1 \\
+11 / 2 \\
+31 / 2 \\
+1 / 2 \\
-1 / 2 \\
\left(+\frac{1}{1}\right) \\
-11 / 2 \\
+2^{1} / 2\end{array}$ & $\begin{array}{l}\text { Grün } \\
\text { Appel } \\
\text { Schwalb } \\
\text { Pess } \\
\text { Veit } \\
\text { Schneider } \\
\text { Reidberge } \\
\text { Nürnberge } \\
\text { Herpel } \\
\text { Grauda }\end{array}$ \\
\hline 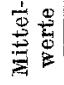 & 4,2 & 5 & 6,6 & 0,98 & $+0,7$ & $+0,9$ & $+0,75$ & $+0,3$ & +1 & $+0,6$ & $+0,3$ & $+0,8$ & \\
\hline
\end{tabular}

Von Bedeutung für die Gerinnungszeit erwies sich jetzt die Zeitspanne der Blutentnahme nach der Injektion, und zwar schien uns der Einfluß des Präparates nach 30 Minuten post injectionem am günstigsten zu sein. Zusammenfassend ergab sich, daß in den meisten der untersuchten Fälle (9 von 11) sich schon nach der ersten Injektion die Gerinnung in besehleunigendem Sinne dokumentierte, und zwar im Durchschnitt um fast eine Minute. 'Eine erhöhte Wirkung des Gerinnungsvermögens durch die $Z a h l$ der Spritzen, wenn solche nur in Abständen von einer Stunde gegeben wurden, war nicht festzustellen. Wahrscheinlich spielen hier Faktoren eine Rolle, wie wir sie im experimentellen Teil der Arbeit beobachten konnten, wonach die Menge der applizierten Chemikalien gewöhnlich keinen steigernden Finflu $B$ ausübt.

Was nun das Wesen der Gerinnungsänderung anlangt, so soll eine spätere Veröffentlichung gemeinsam mit Oppenheimer Aufklärung darüber verschaffen. Ob es sich um rein fermentative oder physikalisch- 
chemische Vorgänge unter dem Einfluß der Proteinkörper handelt, muß noch offen bleiben. Uns kam es heute nur auf die rein praktische Szite des Problems an. Vorstellen könnte man sich, daß es sich um eine paptonähnliche Wirkung handelt: Uns scheint aber am einleuchtendsten, daß eine Beeinflussung der Thrombocyten, deren hohe Bedoutung besonders von Bürker erkannt wurde, wesentliche Momente abgibt. Diesbezügliche Versuche sind bei uns im Gange. Auch H. Freund nimmit an, daß die parenteral eingeführten Proteine gar nicht direkt wirksam werden, sondern erst die gebildeten körpereigenen Eiweißspaltprodukte, wobei auch er an einen Zerfall der emplindlichen Blutplättchen denkt.

Wenn wir in Kürze einige unserer Ergebnisse zusammenfassend herausgreifen wollen, so verdienen folgende Punkte nochmals besonders hervorgehoben zu werden.

Die vergleichenden Gerinnungsversuche waren nur möglich mit dem von uns benutzten Apparat von Bürker, der ganz exakt arbeitet unter stets gleichen Bədingungen. Die Technik der Bedienung muß einwandfrei beherrscht werden. Die normale Blutgerinnung ist für den Menschen eine ziemlich konstante und schwankt um fünf Minuten.

Die Eiweißkörper (und eine Roihe anderer Chemikalien) üben einen starken Einfluß auf die Gerinnungsbereitschaft des Blutes aus. Wird die Blutgerinnung geprüft gegen unverdiunnte Proteinkörper (Caseosan und Aolan), so offenbart sich eine deutliche Hemmung; dagegen tritt ein Umschlag nach der entgegengesetzten Seite ein mit steigender Verdünnung der Präparate, so daß bei $1 / 10^{-}$-Verdünnung eine Beschleunigung der Hämostase festzustellen ist. Individuell wirken die Eiweißkörper verschieden, jedoch bleibt auch dabei die obige Gesetzmäßigkeit bastehen. Werden Eiweißkörper oder Placenta-Opton dem Organismus intravenös oder intramuskulär einverleibt, so tritt eine deutliche Änderung des Grinnungsspiegels zutage.

Unsere Untersuchungen eröffnen neue Gasichtspunkte über die Wirkungsweise der Proteinkörper. Um nur ein praktisches Anwendungsgebiet herauszugreifen, so dürften sie z. B. vor opærativen Eingriffen appliziert, die Blutgerinnungsbereitschaft heben und gleichzeitig leistungssteigernd auf den ganzen Körper wirken und damit dessen Widerstandsfähigkeit erhöhen. Klinische Erfahrungen müssen jetzt diese prophylaktische Wirkungsweise beweisen.

\section{Literatur.}

Bürker, Pflügers Arch. f. d. ges. Physiol. 102. 1904. - Bürker, ebenda 149. Bürker, Tigerstedts Handb. d. physiol. Methodik 2, Abt. I u. V. - Esch, Zentralbl. f. Gynäkol. 1920, Nr. 31. - Freund, H., Məd. Klin. 1920, Nr. 17. - v. Jaschke 
332 R. Salomon u. E. Vey : Der Einfluß von Proteinkörpern auf die Blutgerinnung.

und Siegel, Münch. med. Wochenschr. 1920, Nr. 21. -- Klingmüller, Dtsch. med. Wochenschr, 1917, Nr. 41 u. Münch. med. Wochenschr. 1919, Nr. 33. - Lindig, Münch. med. Wochenschr. 1919, Nr. 33. - Morawitz, Handb. d. biochem. Arbeitsmethoden 5, Teil 1. .- Müler, E. F., Dtsch. med. Wochenschr. 1919, Nr. 12 u. Med. Klinik 1918, Nr. 18 u. 28. - Opitz, Münch. med. Wochenschr. 1920, Nr. I. Reinewald, $P$, Inaug.-Diss. Marburg 1922, - Salomon, Rud., Münch. med. Wochen-

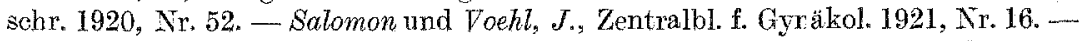
Seitz, A., Klin. Wochenschr. 1922. - Starkenstein, Münch. med. Wochenschr. 1919, Nr. 8. - Voehl, Arch. f. Gynäkol. 114. 1921. - Weichardt, Münch. med. Wochenschr. 1918, Nr. 22; 1919, Nr. 11; 1920, Nr.4. - Zimmer, Münch. med. Wochenschr. 1921, Nr. 18; Berl. klin. Wochenschr. 1921, Nr. 20, 43 u. 44. 